\section{Colostomy as a Bridge to Definitive Pediatric Surgical Care: A Sub-Saharan African Experience}

There is a paucity of data regarding the true burden of surgical conditions that affect children living in sub-Saharan Africa. Available data, however, points to a wide variety of surgical conditions, with the majority falling into three major diagnostic categories, injuries, congenital anomalies, and surgical infections. ${ }^{1}$ In Africa, children constitute more than half the population. ${ }^{2}$ Despite high patient volume, very few dedicated pediatric surgeons are available, with only one pediatric surgeon to approximately two million children in Africa, compared with 1:100,000 in North America. ${ }^{3}$ Owing to this dearth of pediatric surgical workforce, surgical disease in children is not often managed, and if managed, is predominantly managed by a general surgeon who may lack familiarity with complex pediatric surgical procedures.

An ostomy is the deliberate creation of an opening that communicates between the gastrointestinal tract and the exterior. In the pediatric population, there are a number of acquired and congenital conditions that require ostomy formation, either as a temporary or permanent strategy, such as colonic obstruction, inflammatory bowel disease, traumatic disruption of the gastrointestinal tract, gastrointestinal malignancy, necrotizing enterocolitis, colonic atresia, Hirschsprung's disease, or anorectal malformation.

There is little information in the literature regarding the clinical indications for pediatric patients receiving colostomies, complications associated with colostomies, and colostomy reversal rates in sub-Saharan Africa. Therefore, the aim of this study is to describe the characteristics and outcomes of patients receiving

Address correspondence and reprint requests to Anthony Charles M.D., M.P.H., F.A.C.S., Department of Surgery, University of North Carolina, 4008 Burnett Womack Building, CB 7228. E-mail: anthchar@med.unc.edu. colostomies in a sub-Saharan African setting. By understanding this, we may be able to identify barriers to optimal care of the pediatric surgical patient in subSaharan Africa.

This is a retrospective database analysis of pediatric surgery patients at Kamuzu Central Hospital $(\mathrm{KCH})$ in Lilongwe, Malawi ${ }^{4}$ from February 2012 and June 2015. This database includes patients aged $\leq 18$ years with a surgical diagnosis, admitted to a surgical ward, or that had surgical consultation. Our analysis focused on patients with procedure codes indicating colostomy, colostomy revision, or reversal. Also, patients with a diagnosis that will otherwise mandate a colostomy as part of the management strategy, such as patients with anorectal malformations or Hirschsprung's disease, were included. Data analysis was performed using STATAv14 and Microsoft Excel software. Both the University of North Carolina Institutional Review Board and the National Health Sciences Research Committee of Malawi (NHSRC) approved this study.

There were 4348 patients in the pediatric surgery database, of which 1573 (36.2\%) patients had operative intervention; a large portion of the remaining patients comprised those with traumatic injury. A total of 115 children met inclusion criteria for this study. Of these, $71(61.7 \%)$ were male, with an overall mean age of $2.3 \pm 2.9$ years. There were 55 $(47.8 \%)$ patients that received an operation during their admission. The mean number of days from admission to surgery was $13.5 \pm 14.5$ days and the average length of stay was $14 \pm 12.6$ days. No deaths occurred in any of the patients of our study, compared with the overall pediatric surgery database with a mortality of 0.4 per cent.

Of the 55 patients who had an operative intervention, the most common operation was colostomy, 27 (49.1\%). The second most common operation was exploratory laparotomy, eight (14.5\%) (Table 1). Analysis of the admission diagnoses of patients receiving a colostomy revealed congenital disease as the most common indication, specifically, anorectal 
malformation, 12 (44.4\%), followed by Hirschsprung's disease, seven (25.9\%) (Table 2). However, 60 (52.2\%) admitted patients did not receive any operative intervention. Of these, $44(73.3 \%)$ patients had congenital disease $-15(25 \%)$ patients with anorectal malformations and $29(48.3 \%)$ with Hirschsprung's disease.

During the study period, of the total 115 patients, 83 patients were seen at $\mathrm{KCH}$ with a congenital diagnosis, consisting of either anorectal malformation or Hirschsprung's disease. The 83 patients were comprised 39 patients with congenital disease that received an operation and 44 patients with congenital

TABle 1. Operations Performed for Patients with Colostomy-Related Admissions and with Congenital Disease (Hirschsprung's Disease or Anorectal Malformation); EUA = exam under anesthesia

\begin{tabular}{lcc}
\hline \multicolumn{3}{c}{ Operations Performed } \\
\hline & Number of Patients & Percentage \\
\hline Colostomy & 27 & 49.1 \\
Exploratory laparotomy & 8 & 14.5 \\
Biopsy & 7 & 12.7 \\
Anal dilation & 3 & 5.5 \\
Other & 3 & 5.5 \\
Colostomy takedown & 2 & 2.6 \\
Pull through & 2 & 2.6 \\
Urologic procedure & 2 & 2.9 \\
EUA & 1 & 1.8 \\
\hline
\end{tabular}

TABLE 2. Admission Diagnoses of Patients that Had a Recorded Operation Code of Colostomy

Admission Diagnoses of Patients Receiving a Colostomy

\begin{tabular}{lcc}
\hline & Number of Patients & Percentage \\
\hline Anorectal malformation & 12 & 44.4 \\
Hirschsprung's & 7 & 25.9 \\
Bowel obstruction & 2 & 7.4 \\
Other & 2 & 7.4 \\
Fistula & 1 & 3.7 \\
Acute abdomen & 1 & 3.7 \\
Abdominal mass & 1 & 3.7 \\
Colostomy revision & 1 & 3.7 \\
\hline
\end{tabular}

disease that did not receive an operation. The rate of colostomy was smaller in patients with congenital disease when compared with those without, 19 of 83 $(22.9 \%)$ versus 8 of $32(25 \%)$, respectively $(P=$ 0.8 , although not statistically significant. We detected no statistically significant differences in overall surgery rates, 39 of $83(47 \%)$ versus 16 of 32 $(50 \%), P=0.7$, in those with and without an underlying congenital abnormality, respectively. Furthermore, patients with congenital diseases compared with those without congenital diseases had increased elapsed time between admission to surgery, $15.1 \pm$ 15.8 days versus $9.8 \pm 10.2$ days $(P=0.2)$, respectively, though not statistically significant, as well as significantly increased elapsed time between surgery and discharge, $9.3 \pm 8.9$ days versus $5.1 \pm$ 3.6 days, respectively $(P=0.03)$. There was no significant difference in overall hospital length of stay, $(P=0.3)$ (Table 3$)$.

Notably, the posthospitalization follow-up plan for most patients appeared to be lacking. Although 27 patients received a colostomy and 16 patients were admitted with a colostomy diagnosis, no patients were listed as having any discharge needs, including no indication for any ostomy-related needs upon discharge. Furthermore, only $32(27.8 \%)$ patients returned for a follow-up clinic visit, and of these, only seven $(21.9 \%)$ were patients that had received a colostomy. Finally, during our study period, there were only four patients who returned for reversal of their colostomy, with two colostomy takedowns and two pull-through procedures, with an average of $261.3 \pm 288.1$ days till reversal (range 36 to 654 days).

Based on our study of a pediatric surgery database at $\mathrm{KCH}$ in central Malawi, we show that the majority of pediatric patients receiving a colostomy had a congenital anomaly such as anorectal malformation and Hirschsprung's disease as the indication for the procedure. However, there is a large portion of these children with congenital disease that are being seen but are not receiving at least a bridging colostomy. On closer analysis of the subset of patients with congenital anomalies, those that underwent an operative

Table 3. Outcome Based on Type of Diagnosis - Congenital Disease Versus Noncongenital Disease

\begin{tabular}{lccc}
\hline \multicolumn{3}{c}{ Outcome Based on Type of Diagnosis } & \\
\hline Operative intervention & Congenital & Noncongenital & $P$ Value \\
Colostomy & $39(47 \%)$ & $16(50 \%)$ & 0.7 \\
Days to surgery & $19(22.9 \%)$ & $8(25 \%)$ & 0.8 \\
Days from surgery to discharge & $15.1 \pm 15.8$ & $9.8 \pm 10.2$ & 0.2 \\
Length of stay (days) & $9.3 \pm 8.9$ & $5.1 \pm 3.6$ & 0.03 \\
\hline
\end{tabular}


intervention were still less likely to have a colostomy performed when compared with the noncongenital cohort. These patients were undergoing less invasive procedures such as anal dilation. It appears that the capacity for the management of congenital diseases, with temporizing procedures such as colostomy formation, is lacking in central Malawi, and there is a great need for the use of colostomy as a bridge therapy to definitive care, particularly in children with congenital anomalies. In this setting, initial treatment with colostomy may be a safe procedure that can be completed confidently by a general surgeon. However, this must be viewed as a temporary solution (bridge) to definitive therapy (destination) at $\mathrm{KCH}$ or elsewhere. There must be increased efforts to improve education and resources so that colostomies are reversed for more definitive procedures. Furthermore, the importance of parent or guardian education before leaving the hospital as well as emphasis on the importance of posthospitalization follow-up is crucial to minimize morbidity and mortality associated with colostomy formation. In sub-Saharan Africa, the minimum bar for the care of the pediatric surgical patients has yet to be met. Good data are needed to inform good public health policy. We believe that investments in the training of general surgeons and other nonphysician clinicians in the provision of basic pediatric surgical procedures is imperative, if we are to improve infant and childhood mortality as set forth by the United Nations Millennium Development Goals.
Mansi Shah, M.D.

Jared Gallaher, M.D.

Department of Surgery

University of North Carolina

Chapel Hill, North Carolina

Nelson Msiska, C.O.

Kamuzu Central Hospital

Lilongwe, Malawi

Sean E. McLean, M.D.

Anthony G. Charles, M.D., M.P.H.

Department of Surgery

University of North Carolina

Chapel Hill, North Carolina

\section{REFERENCES}

1. Ozgediz D, Poenaru D. The burden of pediatric surgical conditions in low and middle income countries: A call to action. J Pediatr Surg 2012;47:2305-11.

2. Center for Human Rights. Children's Rights in Africa. Available at: http://www.chr.up.ac.za/index.php/ahrc-courses/childrensrights-in-africa.html. Accessed December 3, 2015.

3. Mshelbwala PM, Nwomeh BC. Paediatric surgery specialty and its relevance to Africa. In: Ameh E, Bickler S, Lakhoo K, et al., eds. Paediatric Surgery: A Comprehensive Text For Africa. Seattle: Global HELP Organization, 2011. pp 3-7. Available at: www. global-health.org. Accessed December 3, 2015.

4. Kendig CE, Samuel JC, Varela C, et al. Pediatric surgical care in Lilongwe, Malawi: outcomes and opportunities for improvement. J Trop Pediatr 2014;60:352-7. 